\title{
Notas Sobre las Dalmas de México
}

\section{BIBLIOTECA DEL ThSTRUUTO DE BIOLOGIA \\ Por E. HERNANDEZ XOLOCOTZI}

Como punto de partida para el estudio de las palmas de México, es interesante saber las especies que hasta la fecha han sido colectadas. A continuación se anota la lista de especies que ocurren en México en forma silvestre según Dahlgren (Dahlgren, B. E. Field Museum of Natural History. Botanical Series. vol. XIV. Index of American Palms. 1936).

1. Acoelorraphe pimo (Becc.) Bartlett. 2. Acrocomia mexicana Karw. 3. Bactris acuminata Liebm. 4. B. boculifera Karw. ex Mart. 5. B. mexicana Mart. 6. Brahea Berlandieri Bartlett. 7 B. calcarea Liebm. ex Mart. 8. B. Conzattii Bartlett 9. B. dulcis (HBK) Mart. 10. B. dulcis var. montereyensis Becc. 11. Calyptrogyne Gieslireghtiana (Linden \& Wendl.) Wendl. 12. Chamaedorea affinis Liebm. 13. C. alternans Wend1. 14. C. atrovirens Wendl. 15. C. lirevifrons Wend1. 16. C. cataractarum Liebm. ex Mart. 17. C. concolor Mart. 18. C. desmoncoides Wendl. 19. C. elatior Mart. 20. C. glaucifolia Wendl. 21. C. graminifolia Wend1. 22. C. humilis (Liebm.) Mart. 23. C. Karwinskyana Wendl. 24. C. Klotzchiana Wendl. 25. C. Liebmannii Mart. 26. C. Martiana Wendl. 27. C. microspadix Burret 28. C. monostachys Burret 29. C. montana Liebm. 30. C. oblongata Mart. 31. C. oreophila Liebm. ex Mart. 32. C. paradoxa Wendl. 33. C. pochutlensis Liebm. 34. C. Pringlei Wats. 35. C. pygmaea Wendl. 36. C. radicalis Mart. 37. C. resinifera Wendl. 38. C. rigida Wendl. ex Dammer. 39. C. scandens Liebm, ex Mart. 40. C. Schiedeana Mart. 41. C. stolonifera Wendl. ex Hook. 42. C. renella Wendl. 43. C. tepejilote Liebm. 44. C. Wendlandiana (Oerst.) Hemsl. 45. Coccothrinax a:gentea (Lodd.) Sargent. 46. Collinia elegans (Mart.) Lizbm. 46. C. elegans var. angustifolia Martens \& Galeotti. 47. Cryosophila argentea Bartlett. 48. C. nana (HBK) 
Blume ex Jacks. 49. Desmoncus chinantlensis Liebm. 50. Eleuthe ropetalum Ernesti-Augusti (Wendl.) Oerst. 51. E. Sartorii (Liebm.) Oerst. 52. E. Sartorii var. conferta (Wendl.) Burret. 53. Erythea aculeata Brandegee. 54. E. armata Watson. 55. E. Brandegeei Purpus. 56. E. Brandegeei var. spiralis Jones. 57. E. edulis (Wendi.) Watson. 58. E. elegans Franccschi ex Becc. 59. E. loretensis Jones 60. E. Roezlii (Linden) Becc. 61. Geonoma magnifica Linden \& Wendl. 62. G. oxycatpa Niart. 63. G. Preussii Burret. 64. Hexopetion mexicanum (Liebm.) Burret. 65. Orbignya cohune (Mart.) Dahlgren. 65. Reinhartia elegans Liebm. 67. Sabal Dugessi S. Wats. ex Bailey 68. S. japa Wright. 69. S. mayarum Bartlett. 70. S. mexicana Mart. 71. S. Rosei (Cook) Becc. 72. S. texana (Cook) Becc. 73. S. urcsana Trelease. 74. Scheelea gomphococca (Miart.) Burret. 75. S. Liebmannii Becc. 76. S. Preussii Burret. "77. Thrinax parviflora Swartz. 78 T. microcarpa Sarg. 79. Washingtonia filifera (Linden) Wendl. 80. W. robusta var. gracilis (Parish) Becc. 81. W. sonorae Watson.

De la lista anterior se desprende que la familia de las palmas está representada a la fecha en México por 19 géneros que incluyen 76 especies y 5 varicdadcs. Pero quienquiera que se interese en este grupo de nuestra flora pronto se dará cuenta de que nuestro conocimiento del mismo es sumamente fragmentario. A la fecha todavía no se sabe la idenúdad botánica del "coquito de aceite" de la costa del Pacífico, y cualquiera colección, por pequeña que sea, probablemente incluirá nuevas especies.

* NOTA: Scheelea Preussii Burret se había encontrado solamente en Guatemala, pero en una visita a Chiapas pude comprobar su distribución en México en una franja de la costa aproximadamente de Pijijiapan a Suchiate en el Estado de Chiapas. 